\title{
THz source based on laser mixing
}

\section{Gliese, Ulrik Bo}

\section{Published in:}

Proc. of the IEEE MTT-S \& LEOS Topical Meeting on Microwave Photonics

Publication date:

1997

\section{Document Version}

Publisher's PDF, also known as Version of record

Link back to DTU Orbit

Citation (APA):

Gliese, U. B. (1997). THz source based on laser mixing. In Proc. of the IEEE MTT-S \& LEOS Topical Meeting on Microwave Photonics (pp. 87-90). IEEE.

\section{General rights}

Copyright and moral rights for the publications made accessible in the public portal are retained by the authors and/or other copyright owners and it is a condition of accessing publications that users recognise and abide by the legal requirements associated with these rights.

- Users may download and print one copy of any publication from the public portal for the purpose of private study or research.

- You may not further distribute the material or use it for any profit-making activity or commercial gain

- You may freely distribute the URL identifying the publication in the public portal

If you believe that this document breaches copyright please contact us providing details, and we will remove access to the work immediately and investigate your claim. 


\title{
THz Source based on Laser Mixing
}

\author{
U. Gliese \\ Center for Broadband Telecommunications, Department of Electromagnetic Systems, \\ Technical University of Denmark, Building 348, DK-2800 Lyngby, Denmark \\ Phone: +45 458814 44, Fax: +45 4593 1634, E-mail: ug@emi.dtu.dk
}

\begin{abstract}
The concept of a $\mathrm{THz}$ source based on laser mixing is presented. A radiated power of $100 \mu \mathrm{W}$ can be generated at 1-3 THz from laser powers of $25 \mathrm{~mW}$ if a photo detector with a bandwidth efficiency product of $360 \mathrm{GHz}$ can be fabricated.
\end{abstract}

\section{Introduction}

Far-infrared (sub-millimetre wave) heterodyne radiometry is of significant importance for astrophysics and atmospheric research [1], [2]. Technology for the frequency range of $300 \mathrm{GHz}$ to approximately $700 \mathrm{GHz}$ is now sufficiently mature to be considered for application in space borne instruments [1]. There is, however, a strong push to go to the 1-3 $\mathrm{THz}$ range [1]-[3].

Apart from the availability of mixers operating at $\mathrm{THz}$ frequencies, there is the even more difficult problem of developing the required local oscillator sources [1] [3]. The output power of solid-state Gunn-diode oscillators and multipliers drop off very rapidly above $700 \mathrm{GHz}[1]-[3]$. Today, the only feasible way to achieve $\mathrm{THz}$ radiation is by use of a far-infrared (FIR) gas laser that is pumped by a $\mathrm{CO}_{2}$ gas laser [1]-[3]. This type of source is, however, quite bulky and heavy and certainly not suitable for space borne systems. Therefore, new techniques and technologies for $\mathrm{THz}$ sources are currently investigated by both ESA and NASA.

Compact, yet efficient, $\mathrm{THz}$ sources can possibly be obtained by the use of coherent optical techniques [4], [5]. The $\mathrm{THz}$ signal is generated by mixing of two laser signals in a suitable mixing element. Compact lasers such as semiconductor lasers or $\mathrm{Nd}: \mathrm{YAG}$ lasers can be used together with fibre-optic technology resulting in $\mathrm{THz}$ sources that may be usable in space borne systems. Such a source has been investigated as part of the ESA program, and the basic concepts are presented in this paper together with a performance estimation.

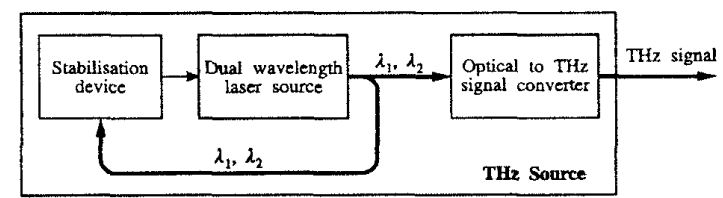

Figure 1: The principal parts of a $\mathrm{THz}$ source based on laser mixing.

\section{THz source principle}

As shown in Fig. 1, any type of $\mathrm{THz}$ source based on laser mixing consists of three distinctive parts:

1. A dual wavelength laser source.

2. A Stabilisation device.

3. An Optical to $\mathrm{THz}$ signal converter.

The dual wavelength laser source generates two optical signals with a frequency offset corresponding to the frequency of the desired $\mathrm{THz}$ signal. A stabilisation device is necessary to ensure the correct frequency offset, to stabilise the frequency and to remove laser phase noise. The $\mathrm{THz}$ signal is generated from the two offset stahilised optical signals by mixing in a suitable optical to $\mathrm{THz}$ signal converter.

\section{Stabilised dual wavelength laser source}

The following laser sources have been identified as possible candidates [4], [5]:

1. Two separate lasers:

a. Semiconductor lasers.

b. Solid state lasers.

2. A dual wavelength laser:

a. Solid state parametric oscillator.

All of these require stabilisation by optical offset phase locking for the reasons described above. 


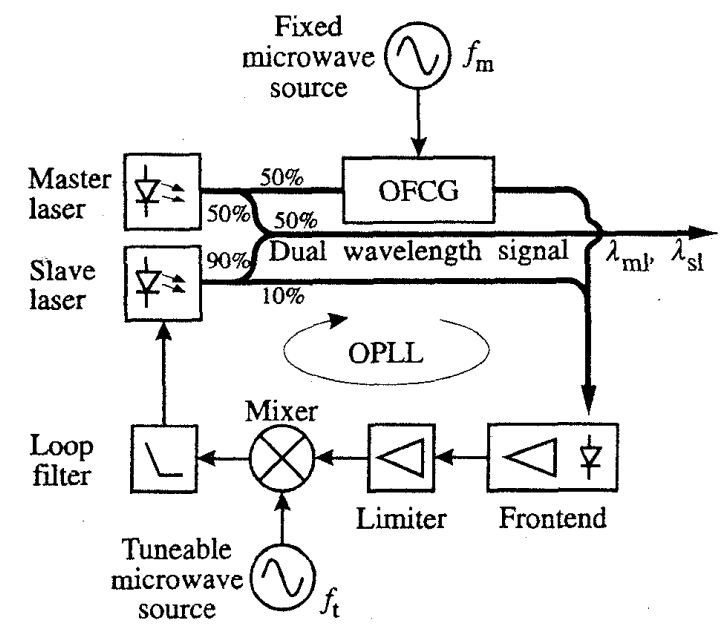

Figure 2: Schematic of the OPLL stabilised dual wavelength laser source.

Semiconductor lasers would be highly desirable for space applications. Commercially available devices, however, require wideband phase locking [6]. In contrast, solid state lasers only require narrowband phase locking [7]. The parametric oscillator has been used with promising results [8]. However, it is far too less developed for space application. At present, the most promising solution seems to be two separate compact Nd:YAG lasers. These are commercially available, are developed to an extend that enable application in space, and only require narrowband phase locking.

The dual wavelength laser source based on two lasers stabilised by an optical phase locked loop (OPLL) is shown in Fig. 2. It consists of a freerunning master laser, a locked slave laser, an optical frequency comb generator (OFCG), a fixed microwave source, an optical frontend, a limiting microwave amplifier chain, a microwave mixer, a tuneable microwave source and a loop filter.

As no $\mathrm{THz}$ reference exists, it is necessary to use harmonic phase locking. The harmonics are generated optically by the OFCG shown in Fig. 3. It consists of a microwave resonant phase modulator placed inside a Fabry-Perot cavity [8]-[10]. As shown, it generates a large set of optical sidebands as harmonics of $f_{\mathrm{m}}$ centred around the frequency of the master laser, $f_{\mathrm{ml}}$. The OFCG can be implemented as a monolithic device, and comb widths of more than $3 \mathrm{THz}$ have been obtained [9]. Harmonic optical phase locking with this type of OFCG has been demonstrated at frequencies as high as $1 \mathrm{THz}[10]$.
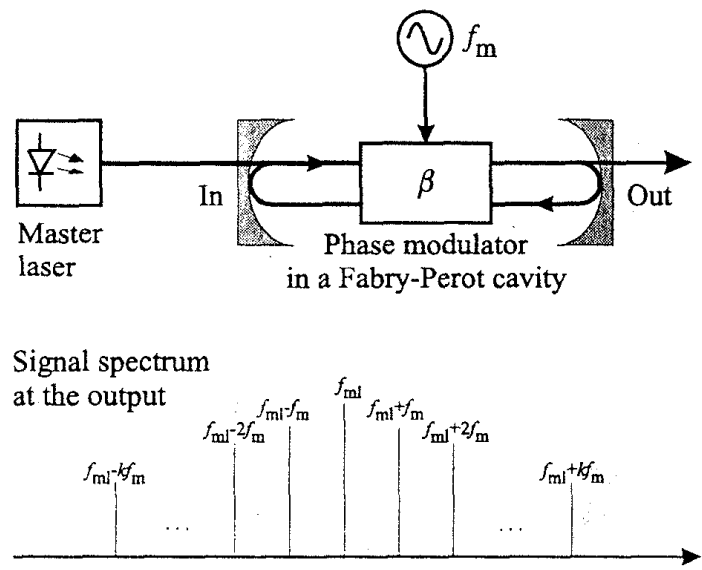

Figure 3: Principle of the optical frequency comb generator.

The optical frequency comb is injected into the photodiode of the frontend together with the signal from the slave laser. The beat signal, between the $k^{\text {th }}$ sideband and the slave laser signal, is amplified and limited in order to obtain a constant power level at the input of the mixer no matter which sideband the slave laser is beating against. In the mixer, the beat signal is compared to the signal from a tuneable microwave source. The resulting phase error difference signal is feed to the slave laser through the loop filter. This forces the slave laser to track the master laser at an offset of $f_{\mathrm{THz}}=k \cdot f_{\mathrm{m}}+f_{\mathrm{t}}$. The offset phase locking ensures a frequency stability, resolution and accuracy that all are equal to $k$ times that of the fixed microwave source at $f_{\mathrm{m}}$. The frequency of the THz offset can be coarse tuned by stepping the slave laser through the OFCG sidebands and fine tuned by means of the tuneable microwave source. Exact determination and control of the $\mathrm{THz}$ offset can be obtained by use of the available OPLL signals and additional and simple control electronics [4], [5].

\section{Optical to THz signal converter}

As the final step, the $\mathrm{THz}$ offset dual wavelength optical signal must be converted into a real $\mathrm{THz}$ signal. The following conversion methods have been identified as possible candidates [4], [5]:

1. Heterodyne conversion in a photo detector: a. Travelling wave PIN photo detector.

b. Schottky MSM photo detector.

c. Photoconductive MSM photo detector. 


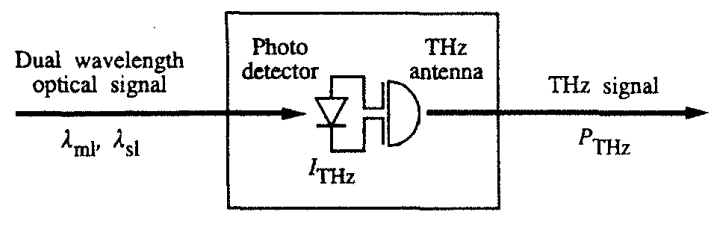

Figure 4: Principle of the optical to $\mathrm{THz}$ signal converter based on heterodyne conversion.

2. Optical parametric conversion in a nonlinear crystal.

3. Optical/THz super heterodyne detection in a Schottky diode.

At present, heterodyne conversion seems to be the most efficient of the three [4], [5]. However, optical parametric conversion may show potential if better non-linear materials are found [4], [5].

The basic principle of the heterodyne conversion concept is illustrated in Fig. 4. The two THz offset phase locked optical signals are coupled into the photo detector by use of a microlensed fibre. This fibre is optimized for the specific detector in use and ensures optimum coupling of light into the device. A THz photo current, $I_{\mathrm{THz}}$, is generated in the photo detector by the heterodyning process. In general, the current depends on the power of the two optical signals and on the external bandwidthefficiency product of the photo detector. The electrodes of the photo detector are coupled directly with a $\mathrm{THz}$ antenna which is driven by the current. This results in a radiated $\mathrm{THz}$ signal with a power of $P_{\mathrm{THz}}$. The power level depends on the photo generated current, the photo detector and antenna parasitics, the antenna impedance and the antenna radiation efficiency.

State of the art results for the bandwidth efficiency product of the three different detector types are shown in Fig. 5 together with analytical calculations as well as detailed simulations. As seen, approximately the same performance can be achieved with all three types. Products of 76,118 and $102 \mathrm{GHz}$ have been acheived with travelling wave (TW) PIN [11], Schottky metal semiconductor metal (S-MSM) [12] and photoconductive (PC) MSM [12] detectors, respectively. It is estimated that the use of quantum structures, such as quantum wires (QW), can significantly improve the results of the PIN [13]. Also the results of the MSM have potential for improvement [14]. Lowering of the device temperature also holds potentials for improvement [4], [5].
S-MSM and PC-MSM finger spacing [ $\mu \mathrm{m}]$

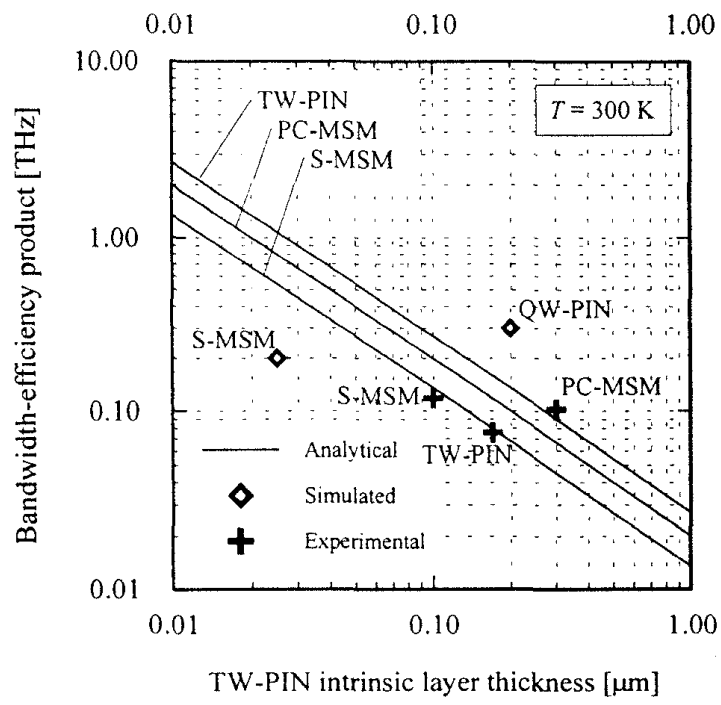

Figure 5: Bandwidth efficiency product of PIN photo detectors versus their intrinsic layer thickness, and of Schottky MSM and Photoconductive MSM photo detectors versus their finger spacing.

\section{Performance estimation}

The performance estimation that is summarised here is based on the results from two ESA research projects. Further details are found in [4], [5].

It is assumed that two compact Nd:YAG lasers with output powers of $25 \mathrm{~mW}$ are used. Further it is assumed that the fibre coupling ratios are as specified in Fig. 2.

A monolithic OFCG is assumed for the sideband generation. It is driven at a frequency, $f_{\mathrm{m}}$, of $19.98 \mathrm{GHz}$, and a modulation index, $\beta$, of approximately $\pi / 2$ is required to cover the $1-3 \mathrm{THz}$ freqency band using the $50^{\text {th }}$ to $150^{\text {th }}$ sideband. This will give a sideband slope efficiency of $5 \mathrm{~dB} / \mathrm{THz}$ which can easily be compensated by the limiter in the OPLL.

A tuning range of $1-20 \mathrm{GHz}$ for $f_{\mathrm{t}}$ will be required to enable continuos tuning between each of the OFCG sidebands.

For the simulated output power in the $150^{\text {th }}$ sideband a carrier to noise density ratio at the input of the OPLL of $100 \mathrm{dBHz}$ has been found based on the data of commercially available lasers, frontend and loop electronics. This gives a loop SNR of more than $30 \mathrm{~dB}$ for loop bandwidths as wide as $1 \mathrm{MHz}$. Such a bandwidth is more than adequate for $\mathrm{Nd}: \mathrm{YAG}$ lasers [7]. 
Table 1: Estimated parameter values.

\begin{tabular}{|l|l|}
\hline Parameter & Value \\
\hline Radiated THz power & $100 \mu \mathrm{W}$ at $1-3 \mathrm{THz}$ \\
\hline Laser output powers & $25 \mathrm{~mW}$ (each) \\
\hline Bandwidth efficiency & $360 \mathrm{GHz}$ \\
\hline OFCG mod. index & $\pi / 2$ at $20 \mathrm{GHz}$ \\
\hline OPLL bandwidth & $<<1 \mathrm{MHz}$ \\
\hline Phase noise & $\sim-90 \mathrm{dBc} / \mathrm{Hz}$ at $1 \mathrm{MHz}$ \\
\hline Frequency accuracy & $\sim 10-30 \mathrm{kHz}$ at $1-3 \mathrm{THz}$ \\
\hline
\end{tabular}

The resulting phase noise and frequency precision of the $\mathrm{THz}$ signal will for 1 and $3 \mathrm{THz}$, respectively, be given by 50 and 150 times the phase noise and frequency precision of the signal at frequency $f_{\mathrm{m}}$.

Besides the OFCG, the implementation of the OPLL is straight forward and can be based on commercially available components. Some development effort is expected for the implementation of an OFCG with the above requirements.

Due to the fibre coupling ratios, the optical power delivered to the converter is $6.25 \mathrm{~mW}$ from the master laser and $11.25 \mathrm{~mW}$ from the slave laser. For the converter with a device integrated antenna, a current to radiated power efficiency of $50 \mathrm{~W} / \mathrm{A}^{2}$ is assumed. To obtain a radiated $\mathrm{THz}$ signal power level of $100 \mu \mathrm{W}$, the photo detector needs a bandwidth efficiency product of $360 \mathrm{GHz}$ for the $3 \mathrm{THz}$ signal. Comparing this to the state of the art results, it is seen that significant development challenges lie ahead for the converter as the photo detector bandwidth efficiency products are a factor of 3-5 too low. However, in this respect, the simulation results of Fig. 5 are encouraging. The main parameter values are summarised in table 1 .

\section{Conclusion}

A promising concept for a compact 1-3 THz source has been presented together with an estimation of the achievable performance and the component requirements. Major parts of the source can be implemented with commercially available components. However, implementation of the required photo detector with a $360 \mathrm{GHz}$ bandwidth efficiency product represents a significant challenge. The source can be expected to deliver $100 \mu \mathrm{W}$ of radiated $\mathrm{THz}$ power with a low phase noise and a high frequency precision. Most importantly, it has potential for use in space borne radiometry instruments.

\section{Acknowledgements}

This work was carried out under ESA, ESTEC contract 140409 and $11653 / 95 / \mathrm{NL} / \mathrm{PB}$.

\section{References}

[1] M.A. Frerking, "Submillimeter source needs for NASA missions," Proceedings of SPIE, vol. 2145, 1994, pp. 222-229.

[2] T.G. Phillips, "THz source requirements for astrophysics receivers," Proceedings of SPIE, vol. 2145, 1994, pp. 230-238.

[3] B.D. Guenther, "Terahertz sources," Proceedings of SPIE, vol. 2145, 1994, pp. 120-129.

[4] U. Gliese et. al., "Feasibility study of optical 1-3 THz signal source," Tech. Report R589, ESTEC contract No. 140409, Tech. Univ. of Denmark, Dept. of Electromagnetic Systems, 1994.

[5] U. Gliese, "THz signal generation by laser mixing," Tech. Report R630, ESTEC Contract No. $11653 / 95 / \mathrm{NL} / \mathrm{PB}$, Tech. Univ. of Denmark, Dept. of Electromagnetic Systems, 1996.

[6] U. Gliese et. al.,

"A wideband heterodyne optical phase locked loop for generation of 3-18 GHz microwave carriers," Photonics Tech. Lett., vol. 4, no. 8, 1992, pp. 936-938.

[7] U. Gliese et. al., "Generation of microwave signals with lasers - Optical offset phase locking of Nd:YAG lasers,"

Tech. Report AR316, Tech. Univ. of Denmark, Dept. of Electromagnetic Systems, 1993.

[8] L.R. Brothers et. al.,

"Terahertz optical frequency comb generation and phase locking of an optical parametric oscillator at $665 \mathrm{GHz}$,"

Optics Lett., vol. 19, no. 4, 1994, pp. 245-247.

[9] M. Kourogi et. al., "A monolithic optical frequency comb generator," Photonics Tech. Lett., vol. 6, no. 2, 1994, pp. 214-217.

[10] K. Nakagawa et. al., "Highly precise $1-\mathrm{THz}$ optical frequency difference measurement of 1.5 um molecular absorption lines," Optics Lett., vol. 20, no. 4, 1995, pp. 410-412.

[11] K.S. Giboney et. al., "Travelling-wave photodetectors with $172-\mathrm{GHz}$ bandwidth and 76-GHz bandwidth efficiency product," Photonics Tech. Lett., vol. 7, no. 4, 1995, pp. 412-414.

[12] S.Y. Chou et. al. "Ultrafast nanoscale metal-semiconductor-metal photodetectors on bulk and low-temperature grown GaAs," Applied Phys. Lett., vol. 61, no. 7, 1992, pp. 819-821.

[13] D.L. Crawford et. al., "Comparison of bulk and quantum wire photodetectors,"

App. Phys. Lett., vol. 58, no. 15, 1991. pp. 1629-1631.

[14] S.Y. Chou et. al.

"Tera-Hertz GaAs metal-semiconductor-metal photodetectors with nanoscale finger spacing and width," Int. Elec. Devices. Meeting Digest, 1991, pp. 745-748. 